\title{
Printed Thermocouple Devices
}

\author{
Sam Duby, Blue Ramsey, David Harrison, Gareth Hay \\ Brunel University, Cooper's Hill Lane, Englefield Green, Surrey, TW20 0JZ, UK \\ Sam.duby@brunel.ac.uk,www.brunel.ac.uk/research/cleaner
}

\begin{abstract}
A novel process for the fabrication of thermocouples using thick-film techniques has been developed. Thermoelectric reactions of $22 \mathrm{\mu V} /{ }^{\circ} \mathrm{C}$ per couple have been observed which are comparable to those of conventional thermocouples. This work outlines the potential for a rapid, lowcost, low temperature manufacturing solution for the production of electrical temperature sensors.
\end{abstract}

\section{Keywords}

Thermocouple, temperature sensors, printed electronics

\section{INTRODUCTION}

Consumer awareness and government legislation have put increasing pressure on manufacturers to invest in and adopt more environmentally-sensitive manufacturing processes. Directives such as WEEE (Waste Electrical and Electronic Equipment) and RoHS (Restrictions of the use of certain Hazardous Substances) have extended producer responsibility and have encouraged manufacturers to regard the impact of their processes on the environment as a primary concern. Research at Brunel University has been done into the application of additive printing processes to the manufacture of electrical components from simple capacitors and resistors $[1,2]$, to more complex integrated filter structures, microwave circuit components and humidity sensors [3]. This work has met with considerable academic and commercial interest. The processes are high-speed, high-volume and offer significant financial and environmental savings over traditional manufacturing processes, both at the production and end-of-life stages.

There are many advantages that directly printed circuitry has over the more conventional printed circuit board. The fact that printing is a high-speed, high-volume process means that there are substantial reductions in production times that lead to large cost reductions. Printing is an additive process that results in substantial environmental benefits over standard techniques. Traditional circuit board manufacture incurs raw materials loses of over $80 \%$ through the waste stream, as an entirely copper coated board is etched away leaving only a small amount of conductive material to form the tracks. Many toxic materials are used in the traditional process, from the photoresists to the etching chemicals, cleaners and fixatives.

Thermocouples have been printed using thick-film processes, which demonstrate a repeatable and stable reaction to temperature and a potential difference per degree temperature change comparable to conventional commercial couples. Results also compare favourably to the thermoelectric performance of other work on novel thermocouple manufac- ture methods, including that reported by Sihai Wen and D.D.L. Chung [4], and Kiyoshi Nogi et al. [5].

This work is continuing to broaden the number of components which can be manufactured by printing processes, reducing material use and manufacturing complexity. It also forms the first step in a more speculative research objective, the development of a low-cost printed thermoelectric generator.

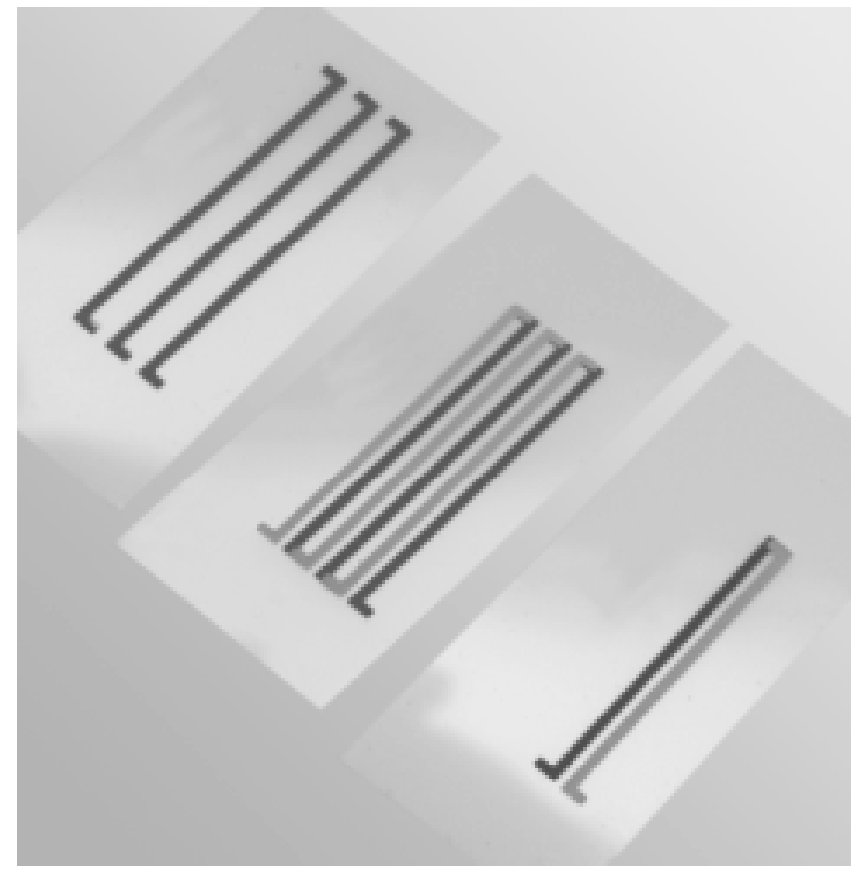

Figure 1. Printed thermocouples $40 \mathrm{~mm}$ long

\section{METHOD}

In order to print a thermocouple, two thermoelectrically active materials with opposite bias need to be processed into inks. In this case, the inks developed consist of metallic particulates suspended in a variety of polyester, cellulose and conductive polyaniline (Panipol $M$ ) resins. The principal functions of the resin vehicle or matrix are to provide suitable flow-characteristics for the ink, to ensure that the final printed track has structural integrity, and to ensure the active particulate is adequately adhered to the polyester substrate. There are a variety of factors that become critical at each stage of the process, and it is the optimisation and refinement of each of these that constitutes the majority of practical work carried out.

In order to maximise the thermoelectric reaction of a printed sample, the proportion of thermoactive material to 
resin must be as large as possible. A small amount of matrix is necessary, as the particulate must remain adequately adhered to the substrate, however minimising the quantity of non-thermoactive material in the final dried printed sample is desirable. In order for an ink to be printable, it needs to have appropriate rheological properties. These characteristics will only be derived from the resin vehicle, and not from the metal particulate itself. As a result, a balance must be sought between formulating an ink with enough resin vehicle to enable printing, and as little resinous material as possible in the final dried printed sample.

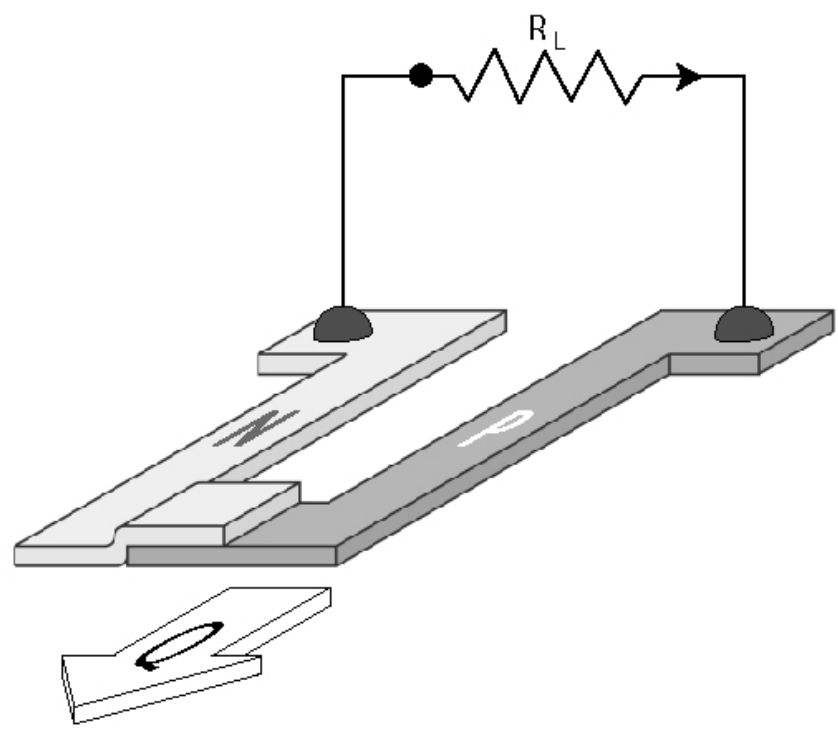

Figure 2. The principle of a printed thermocouple

This balance is addressed through the careful selection of resins with an appropriate molecular weight. Very high molecular weight polymers will lend more viscosity to a stoichiometrically identical solution of lower molecular weight polymers. Only a small quantity of a high molecular weight polymer will need to be added to a suitable solvent to give the resulting solution a relatively high viscosity and flow characteristics that enable printing. On drying, once all of the solvent has evaporated only a small amount of the polymer resin will remain adhering the particulate to the substrate, maintaining the highest possible particulate to matrix proportion.

The image below shows a 1000x magnified photograph of a printed $\mathrm{NiCr}$ track. It can be seen that the particles have been flattened into flake-like form. This is a result of having been ground in a three-roller mill as part of the production process. Flattened particles lie against each other with a greater amount of overlapping surface-area resulting in higher degree of inter-particle contact than nodular or granular particles. This means that the conducting electron does not have as much non-conductive matrix to tunnel through and can relatively easily hop from one particle to the next, facilitating conduction.

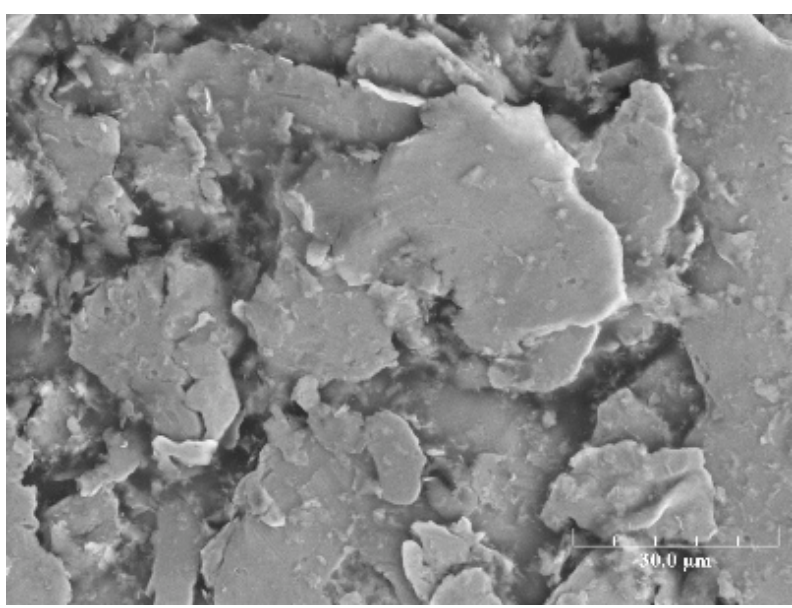

Figure 3. Printed NiCr track $-1000 x$ magnification

Enabling better dispersion of the particulate in the resin resulted in a reduction to the quantity of resin that was needed to create a viable ink. This was done using 'dispersion' or 'wetting' agents. The most effective wetting agent for these purposes was found to be heptanoic acid. The polar nature of this carboxylic acid means that one end of the molecule is repelled by the solvent and attaches to the solid particulate leaving behind a 'tail' that is attracted to the solvent. This is an example of hydrophobic/hydrophilic action and means that the solid particulates are effectively separated from one another and dispersed in the solution.

Various other additives were found to improve the inks. $\mathrm{NaCl}$ for example was outlined as a potentially useful additive in the NiCr based n-type inks because of the fact that chloride ions in solution are very effective at penetrating and destabilising a passive chromium oxide layer. The chemistry of this mechanism has been closely scrutinised and carefully documented because of the corrosive effect that chloride ions have on the chromium in stainless steels. In this case it is inferred that the $\mathrm{NaCl}$ breaks down the protective oxide layer of the chromium, leaving the metal exposed and open to an attractive reaction with a wetting agent, thereby enhancing the wetting mechanism.

A test rig was built to gather thermoelectric data from the printed thermocouple samples. The apparatus was designed such that one end of the thermocouple was held between two air-cooled Peltier modules capable of maintaining a closely controllable temperature of down to $-20^{\circ} \mathrm{C}$, while the other was held between two flat-plate resistance heaters powered by a $240 \mathrm{~V}$ a.c. variable transformer capable of maintaining a stable temperature of between ambient and $170^{\circ} \mathrm{C}$. The sample was only otherwise in contact with the air, ensuring that heat flow was predominately through the printed track with a negligible amount through the polyester substrate. Three mineral-insulated k-type thermocouples probes continually monitored the ambient, cold-junction and hot-junction temperatures through a Thermocouple Data Logger connected to a PC. The data logger was capable of measuring absolute potential difference with a resolution of single microvolts and consequently was also used to monitor the generated emf of the sample. 


\section{RESULTS AND DISCUSSION}

Thermocouples have been printed using a variety of specifically formulated $\mathrm{N}$ and $\mathrm{P}$ type inks. Internal resistances have been driven down to the order of tenths of ohms per square. Experimental data from the test rig gives results for the separate elements that when added together are in close accordance with theoretical data for a complete standard thermocouple (within 5\% variance) [8].

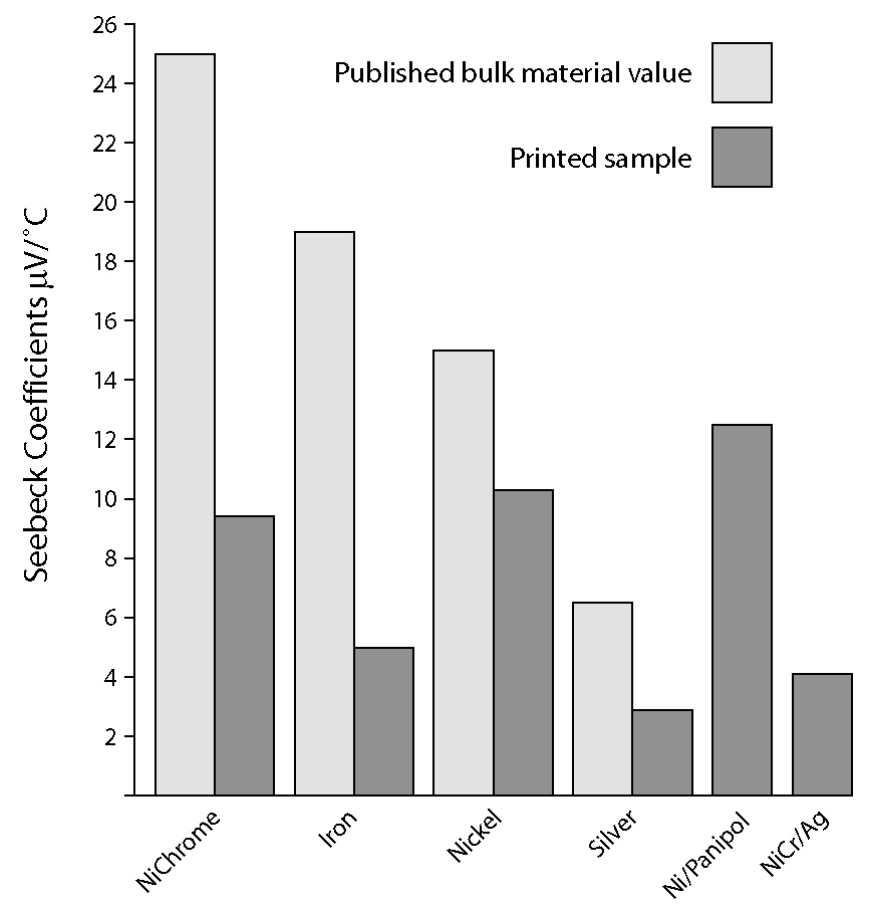

Figure 4. Discrepancy between published bulk material values and those of printed samples
Figure 4 above shows the discrepancy between published bulk material values and those achieved with printed samples. The differences are thought to result from the fact that in contrast to a solid sample, the thermoactive material in an ink is held in a non-electrically conductive matrix. This means that inter-particle conduction can only be achieved with electrons tunneling through the resinous matrix from one conductive particle to the next. Thus hindering the transfer of electrons and hence conduction.

Figure 5 below shows how the electrical reactions of two printed thermoelements compare with those of copper and Nichrome wire, both of which are thermoelements from standard commercially available thermocouples. Data has been gathered over the range of $0-150^{\circ} \mathrm{C}$. The reaction is stable and demonstrates a reasonable hysteresis, typical of standard thermocouple configurations. The best printable thermocouple configurations were found to be a combination of a Nichrome based ink and a Nickel/Panipol blend. This is not a standard thermocouple combination. Data has been gathered that demonstrates that different batches of samples printed with inks of the same formulation perform consistently with a standard deviation of $<0.01$.

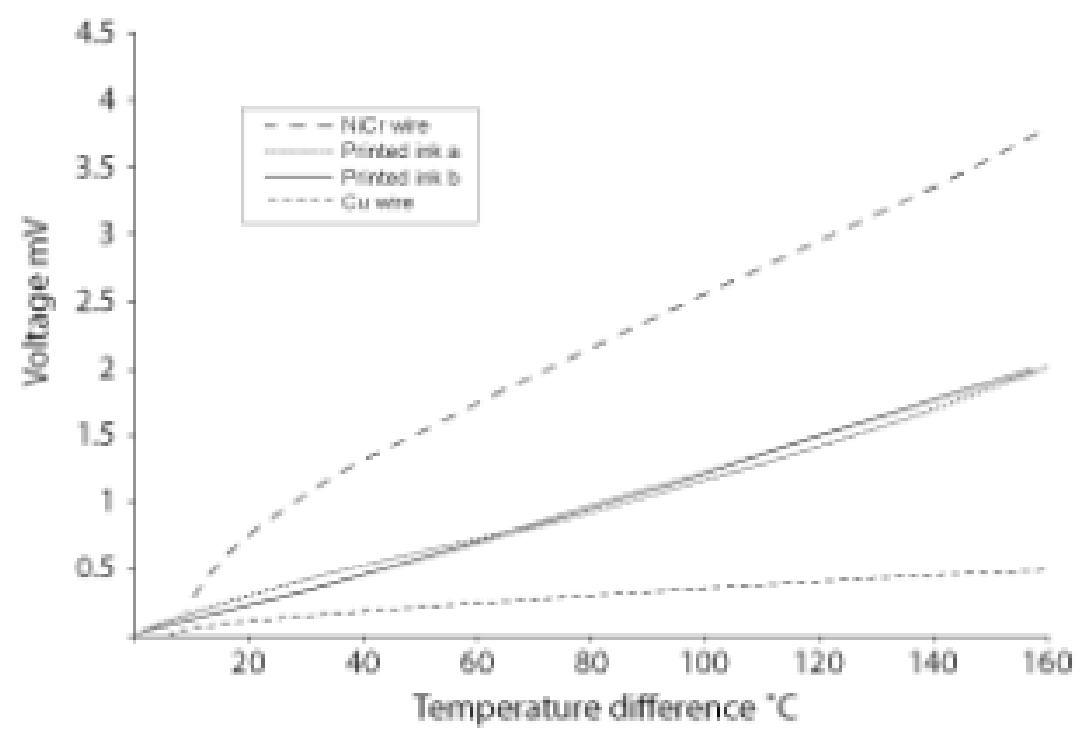

Figure 5. Generated Seebeck voltages of two standard and two printed thermoelements 


\section{CONCLUSIONS}

A novel process for the manufacture of temperature sensing thermocouples has been developed that utilises thick-film techniques. The principal advantage that this process has over traditional thermocouple manufacturing is that of cost. This technique demonstrates the potential application of high-speed, high-volume manufacturing processes.

The physical properties of the materials have defined the temperature range in which these thermocouple sensors will be able to operate. Results show that within the operation temperature range defined by the materials, a reasonable thermoelectric sensitivity is demonstrated. Thermocouples have been manufactured with a thermoelectric sensitivity of $22 \square \mathrm{V} /{ }^{\circ} \mathrm{C}$. This is comparable to commercially available thermocouples.

The experimental results indicate a potential for this approach to offer a rapid, low-cost, low temperature manufacturing process for effective electrical temperature monitoring devices or sensors, overcoming the need to place and solder components and the requirement to 'fire' and laser trim ceramic systems.

One of the more exciting potentials that this work has allowed is the use of new materials as elements in a thermoelectric device. The inclusion of conductive polymers in specific ink formulations had a positive effect on the thermoelectric performance of the device and as such represents an interesting avenue of research.

\section{ACKNOWLEDGMENTS}

This work has been funded by the EPSRC under grant number GR/S08244/01. The authors also wish to acknowledge the valued assistance and support received from Davidson Chemographics.

\section{REFERENCES}

[1] P.S.A Evans, B.J. Ramsey, P.M. Harrey and D.J. Harrison 'Printed analogue filter structures', Electronics Letters, 1999, 35, (4), pp. 306-308

[2] P.M. Harrey, P.S.A. Evans, B.J. Ramsey and D.J. Harrison 'Interdigitated Capacitors by Offset Lithography', Journal of Electronics Manufacture, Vol. 10 No.1, 2000. pp 69-77.

[3] P.M. Harrey, B.J. Ramsey, P.S.A. Evans and D.J. Harrison 'Capacitative-type humidity sensors fabricated using the offset lithographic printing process', Sensors and Actuators. B6361 (2002) 1-7.

[4] Sihai Wen and D.D.L. Chung 'Cement-based thermocouples', Cement and Concrete Research, 2001, 30, pp. 507-510

[5] Kiyoshi Nogi, Takuji Kita and Xiang-Qun Yan 'Production of iron-disilicide thermoelectric devices and thermoelectric module by the slip casting method', Materials Science and Engineering, 2001, A307, pp. 129-133

[6] V.I. Kayadanov and T.R. Ohno 'Process Development and Basic Studies of Electrochemically Deposited CdTe-Based Solar Cells', Annual Technical Report -Colorado School of Mines, 1999, pp. 11-12

[7] B. Yang, J.L. Liu, K.L. Wang and G. Chen 'Simultaneous measurements of Seebeck coefficient and thermal conductivity across superlattice', Applied Physics Letters, 2002, Vol. 80, No. 10, pp 1758-1761

[8] 'Guide to Thermocouple and Resistance Thermometry',TC Limited, Issue 6.0 